\title{
NANOSCALE DEGRADATION MODELS FOR SEMICONDUCTOR, DIELECTRIC AND OXIDE PARTS IN THE DEVICES INCLUDED IN FUNCTIONAL SYSTEMS IN NPP
}

\author{
Angel Popov \\ Sofia University "St. Kliment Ohridski", \\ Faculty of Physics, \\ 5, James Bourchier Blvd, 1164 Sofia, Bulgaria, \\ e-mail: angelsashev@gmail.com
}

\begin{abstract}
In our recent investigation [1] on red GaP LEDs we reported that the decrease of quantum efficiency after aging is accompanied by a change of the ideality coefficient $\beta$ of $(I-U)$ characteristics $\left(I=I_{0} \exp q U / \beta k T\right)$ and the change of the slope of capacitance-voltage $(C-U)$ characteristics. In the latter we have observed a maximum after the dependence $U \sim C^{-1 / 2}$ has changed to $\sim C^{-1 / 3}$.

Keywords: current-voltage characteristics of LED, ideality coefficient $\beta$ of $(I-U)$ characteristics, slope of capacitance-voltage $(C-U)$ characteristics of LED, model of shunting regions of p-n junction in LED, contact resistance $R_{k}$ of "semiconductor-metal" structures, polarizing mechanism forming conductive cords in dielectric structures, occurrence of a double injection in dielectric structures.
\end{abstract}

\section{FUNCTIONAL SEMICONDUCTOR DEVICES WITH ACTIVE BARRIER STRUCTURES}

Two groups, A and B, of LEDs are investigated under identical testing conditions. The difference between these groups are only different crystallization velocities in the LPE process. For group B the crystallization velocity is five times higher than for group A. The change in crystallization velocity changes the structure defect density in the p-layer and in the region of the p-n junction. In this way one can compare the LED characteristics with different densities of structural defects prepared under otherwise equal conditions.

DOI: 10.7546/EngSci.LVII.20.03.01

Engineering Sciences, LVII, 2020, No. 3 
Two new groups C and D were investigated with big amount of copper solved in Ga-epitaxial solution, but the velocities of crystallization processes were different.

For an explanation of the changes of quantum efficiency and electrical characteristics of LEDs after aging we propose a model of shunting regions of p-n junction with higher local conductivity. The general causes of their formation are diffusion and collection of residual impurity atoms on dislocations which are generated during the LPE process. The structural defects like to a linear dislocation line which passes across the p-n junction. The density of dislocations depends on the density of dislocations in the substrate and on the velocity of crystallization. Part of the these generated dislocations has an exit on surface, Fig. 1(a). The rapid diffusion and collection of impurity atoms (more often copper atoms) in the volume of the dislocation take place under the influence of the electromagnetic field at operating time of LEDs. In this way regions of high conductivity are formed and they shunt the p-n junction, Fig. 1(b).

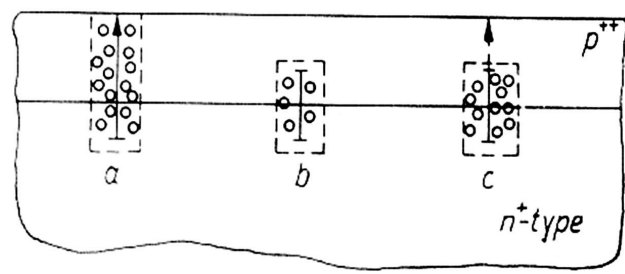

On the other hand, the collection of impurity on the dislocations forms additional tensions in the lattice and results in the lengthening of the closed linear defects including those with an exit on the free surface, Fig. 1(c). This process increases the dark area of the light emitting surface of LEDs.

The change of the slope (Fig. 2) and the appearance of the maximum in $C-U$ characteristics (Fig. 3) are connected with the changes of space charge exclusively, because the shunting regions are formed by collection of the residual impurities on the dislocations as it is shown in Fig. 1. For simplicity the space charge of the p-n junction is approximated by parallelepiped (Fig. 4) in which the shunting region, also with parallelepiped shape, is situated. This approximation permits to account the common balance of the space charge of the reverse biased p-n junction.

As the p-n junction is asymmetric the space charge is located in the higher resistivity $\mathrm{n}^{+}$-region. This concerns the shunting regions also. The space 


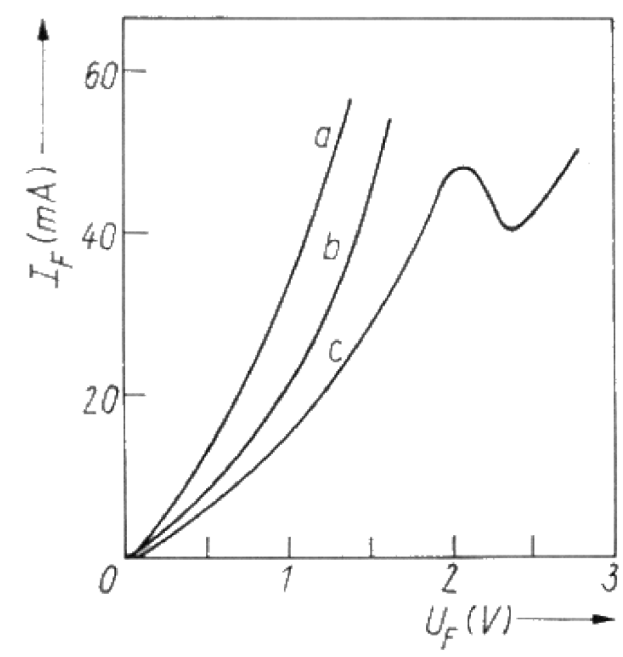

Fig. 2. Current-voltage characteristics of LED from groups $\mathrm{C}$ and D before and after aging $(T=300 \mathrm{~K})$ : (a) before aging $\beta=1.7$; (b) after $500 \mathrm{~h}$ aging $\beta=2.5$; (c) after $1000 \mathrm{~h}$ aging $\beta=3.5$

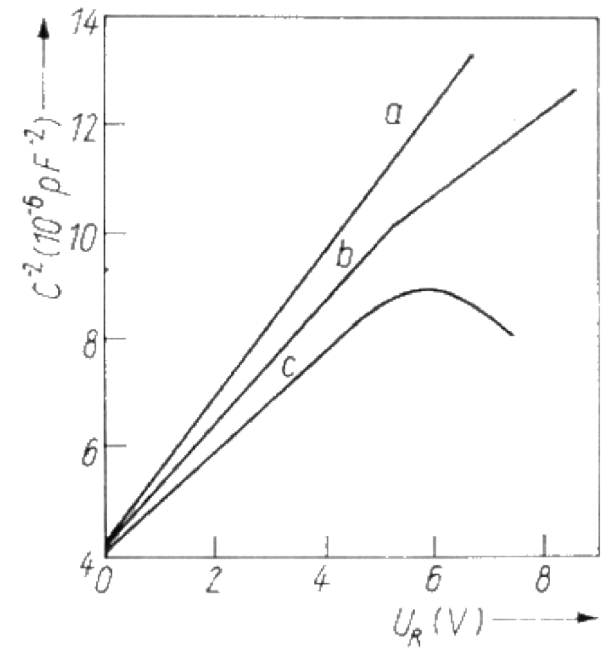

Fig. 3. Capacitance-voltage characteristics of LED before and after aging $(T=300 \mathrm{~K})$ : (a) before aging; (b) after $500 \mathrm{~h}$ aging; (c) after $1000 \mathrm{~h}$ aging

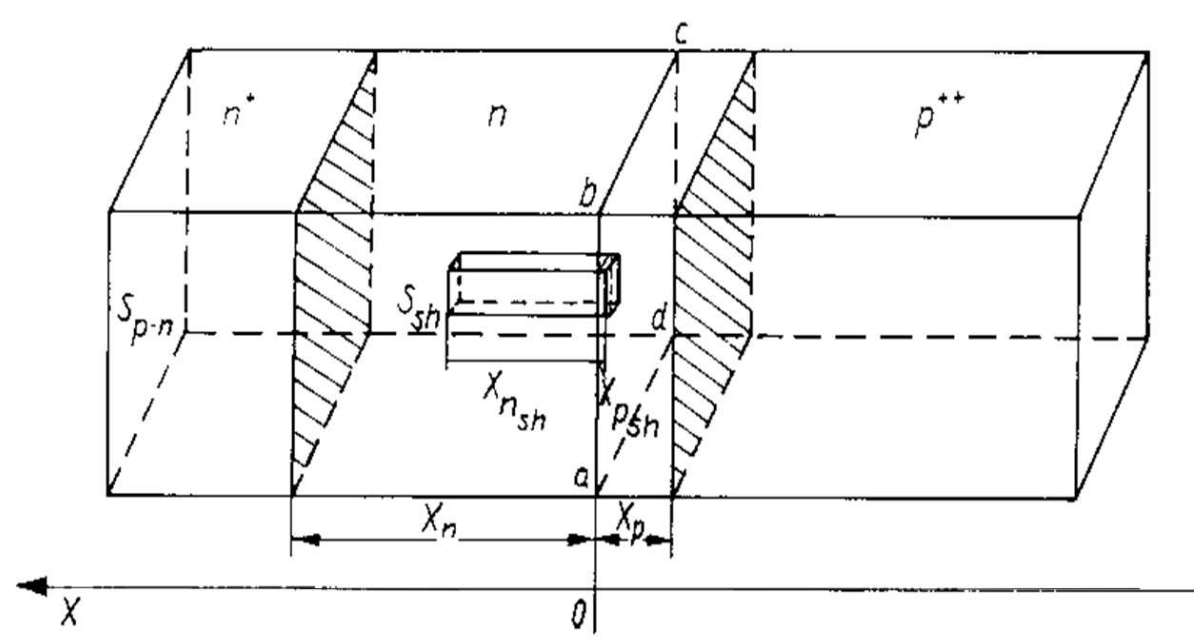

Fig. 4. Model of the p-n junction space charge with shunting region formed on a dislocation without an exit on the p-surface 
charge of the shunting region may be presented at arbitrary coordinate $X$ in the p-n space charge region as:

$$
q(X)=e n_{s h} X_{n s h} S_{s h},
$$

where $n_{s h}$ is the shunting region impurity concentration $\left(n_{s h} \gg n\right.$, the impurity concentration outside the shunting region); $X_{n s h}$ is the space charge width of the shunting region $\left(X_{n s h} \ll X_{n}\right.$, the width of space charge of the p-n junction).

If the space charge width of the shunting region is expressed by the formula for an abrupt p-n junction:

$$
X_{n s h}=\left[\left(2 \varepsilon \varepsilon_{0} U / e n_{s h}\right)\right]^{1 / 2},
$$

the shunting region space charge is

$$
q(X)=S_{s h}\left[2 \varepsilon \varepsilon_{0} e U(X) n_{s h}\right]^{1 / 2} .
$$

If $\mathrm{N}$-shunting regions are included in the space charge region of the $\mathrm{p}-\mathrm{n}$ junction and occupy fraction $\gamma \quad(1>\gamma>0)$ of the area of the p-n junction $\left(\sum S_{s h} / S_{p-n}=\gamma\right)_{N}$, it is possible to determine the average charge density formed by the shunting regions only. This density is equal to:

$$
\varrho_{s h}=\frac{N_{q}(X)}{X_{n} S_{p-n}}=\left(\gamma / X_{n}\right)\left[2 \varepsilon \varepsilon_{0} e U(X) n_{s h}\right]^{1 / 2}=\sigma_{s h}[U(X)]^{1 / 2} .
$$

The total balance of space charge of the p-n junction includes the space charge of shunting regions, the charge on deep levels, as well as the normal p-n junction space charge resulting from shallow impurities. The Poisson equation may be expressed by:

$$
\frac{d^{2} U(X)}{d X^{2}}=\frac{1}{\varepsilon \varepsilon_{0}}\left(\varrho_{p-n}+\varrho_{t}+\varrho_{s h}\right),
$$

where $\varrho_{p-n}$ is the charge density of the non-shunting p-n junction, $\varrho_{t}$ is the charge density of deep level impurities.

The boundary conditions for solving the Poisson equation are derived from the asymmetry of the p-n junction as the space charge is situated practically in the interval $X_{n}>X>0$. Outside of this region we suppose that the semiconductor is neutral, i.e.

$$
\left[\frac{d U(X)}{d X}\right]_{X=X_{n}}=\left[\frac{d U(X)}{d X}\right]_{X=0}=0 .
$$


According to our model the space charge density of the shunting regions is increased considerably after degradation, because the impurity atoms are collected there. Then the following condition takes place:

$$
\sigma_{s h} \gg\left(\sigma_{p-n}+\varrho_{t}\right),
$$

and the Poisson equation becomes very simple. From the analogous solution [2] for the space charge depth it is possible to obtain a formula for the p-n junction capacitance:

$$
C=\varepsilon \varepsilon_{0} S_{p-n}\left[3 U\left(2 \varepsilon \varepsilon_{0} / \sigma_{s h}\right)^{2}\right]^{1 / 3} .
$$

This is valuable conclusion which shows that if the shunting space charge density is higher than the normal $\left(\sigma_{p-n}+\sigma_{t}\right)$ p-n space charge density, a cubic dependence between the capacitance and the reverse bias holds.

The validity of the case $\sigma_{s h} \gg\left(\sigma_{t}+\sigma_{p-n}\right)$ is connected with the rapid decrease of LED efficiency. This situation occurs at the first one or two hundred hours of operating time. After this period of time the decreasing of quantum efficiency becomes more slow in connection with deep level formation. In this case the following condition takes place:

$$
\sigma_{s h}+\sigma_{t} \gg \sigma_{p-n}
$$

When the photoionization of deep impurities in the space charge region increases the space charge in the depletion region changes and respectively leads to an alteration of the barrier capacitance.

For determination of this quantity it is possible to involve a simple degradation model for GaP LEDs, proposed in [3], where is the formula for concentration of nonradiative centers dependimg on operating time. The nonradiative center concentration can be determined by this formula, if their capture crosssection is known. But it must be noted that the clusters may be with different structure and they will form different deep levels, respectively. This process is connected with the changes in the ionized deep impurity concentration, which are expressed:

$$
d N_{n r} / d t=-\varphi \sigma_{n r} N_{n r},
$$

where $N_{n r}$ is the concentration of nonradiative centers; $\sigma_{n r}$ - the capture cross-section of the nonradiative traps; $\varphi$ - the density of the photon flux.

The essence of our method may be presented by following ideas: if the reverse biased p-n junction is irradiated with monochromatic light with photon 
energy $E_{t}<h v<E_{g}$, the only possible mechanism of current carrier generation is that from the impurity level to the valence or conductance bands.

This is valuable conclusion which shows that if the shunting space charge density is higher than the normal $\left(\sigma_{p-n}+\sigma_{t}\right)$ p-n space charge density, a cubic dependence between the capacitance and the reverse bias holds. This fact may explain the abnormal $C-U$ characteristics. The square dependence is valid at low reverse bias, because the depth of total space charge is small and the small amount of shunting regions is included there. In this case the usual Poisson equation is solved.

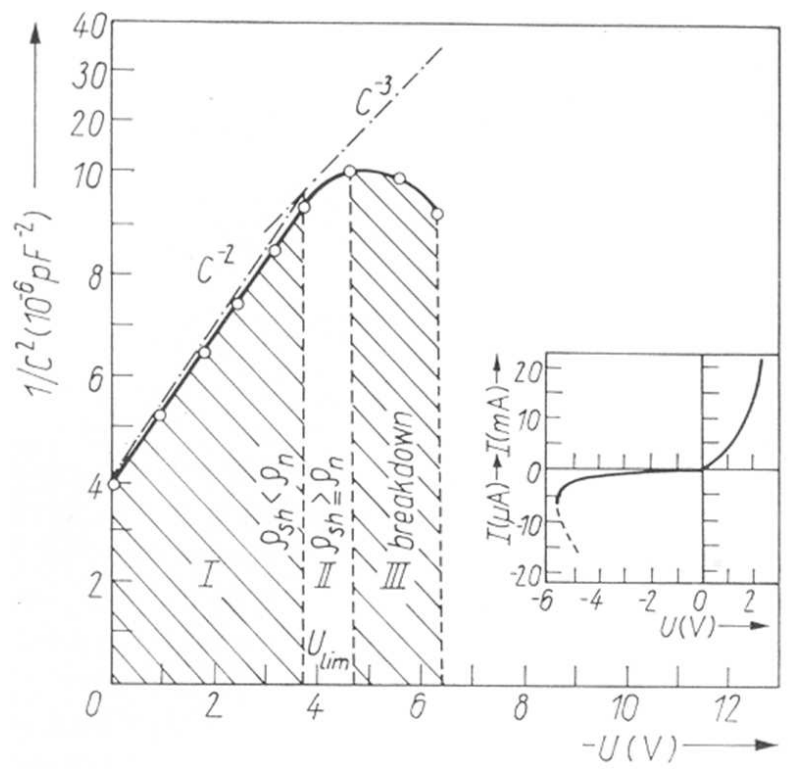

Fig. 5. $C-U$ characteristic of LED after degradation: I region of low space charge density of shunting regions in the space charge region of the p-n junction, II region of high space charge density of shunting regions in the space charge region of $\mathrm{p}-\mathrm{n}$ junction, III region of heating breakdown

The change of the square dependence by the cubic one when $\sigma_{s h} \rightarrow\left(\sigma_{p-n}+\right.$ $\left.\sigma_{t}\right)$ in the region around $U_{\text {lim }}$. The theoretical curve is shown in Fig. 5, which coincides up to the maximum with the experimental one. The maximum and the change of slope after it may be explained by an increasing reverse current, as a big amount of shunting regions is included in the space charge of the $p^{2+}-n^{+}$junction. The average field intensity at the space charge may be presented by formula:

$$
E_{a v}=U / X_{n}=\left(e U N_{\Sigma} / 2 \varepsilon \varepsilon_{0}\right)^{1 / 2} .
$$

The end of breakdown is connected with the heating of the sample. The increase of p-n junction temperature increases the carrier concentration, and the density of static charge due to ionization of deep impurities becomes higher 
also. Thus the slope of the characteristics becomes inversed. The reverse current-voltage characteristic of LEDs after degradation supports this assumption, because the considerable increase of reverse current is observed in the same interval. This fact is connected with the heating breakdown.

The other abnormal phenomenon of LEDs after aging is observed on a forward biased $\mathrm{p}-\mathrm{n}$ junction, where the coefficient $\beta$ of the current-voltage characteristics increases. The change may be explained by the results obtained in [3] for an asymmetric p-n junction. Because the carrier concentration of the one side is higher than on the other, the diode current is carried out by one type of carriers only in large current where $b=\mu_{n} / \mu_{p} ; W$ is the compensation region width; $L$ - the diffusion length of holes; $\mu_{n}, \mu_{p}$-the mobility of electron and holes, respectively.

The application of (12) gives a quantitative estimation of the current, if the n-region of the $p^{++}-n-n^{+}$junction is large or if $W / L>0$. If $W / L \rightarrow 0$, the dependence obtained in [3] transforms to a normal current-voltage characteristic $\left[I-I_{0} \exp (q U / 2 k T)\right]$.

Shunt areas formation and deep levels in sensitive concentrations, influencing on current parameters of diode and transistor, increase the value of the ideality factor as it is shown in Figs 6 and 7. In this case the relation between the width of the spatial charge and diffusion length of carriers increases (becomes bigger than zero) because of length increasing of the spatial charge.

According to [3] the coefficient $\beta$ can be determined by the formula:

$$
B=\frac{2[b+c h(W / L)]}{b+1},
$$

- diffusion of impurities with a small covalent radius into dislocations intersecting p-n junction, and forming shunt fields with a high conductivity;

- as main diffusing element are considered copper atoms, contained in the synthetic semiconductor crystals in high enough concentrations, which are with significantly smaller covalent radius than that of the main atoms of the basic crystal;

- effective coefficient of copper atoms diffusion is defined assuming that the quantity of accumulated impurities is proportional to the operating time and temperature of $\mathrm{p}-\mathrm{n}$ junction.

Radius of the dislocation tube is assumed to be equal to the lattice parameter of $<10 \AA$. The copper diffuses into silicon as positively charged ion. The coefficient of diffusion into n-type and proper silicon is [4]:

$$
D_{\text {int }}=(3.0 \pm 0.3) \times 10^{-4} \exp \left(\frac{0.18 \pm 0.01 \mathrm{eV}}{k_{B} T}\right), \quad \mathrm{cm}^{2} \mathrm{~s}^{-1} .
$$

Engineering Sciences, LVII, 2020, No. 3 


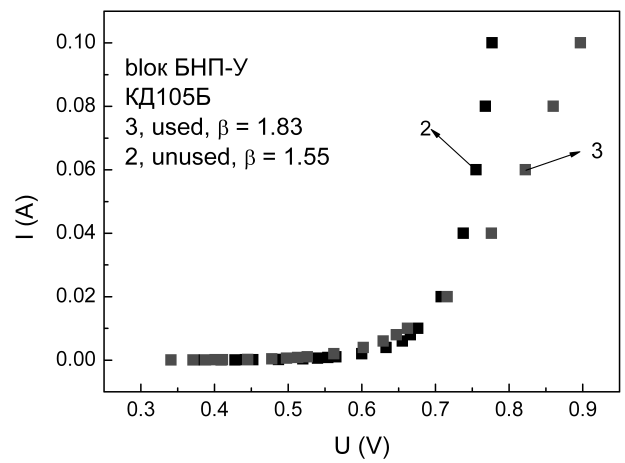

Fig. 6. Factor ideality of unused diode

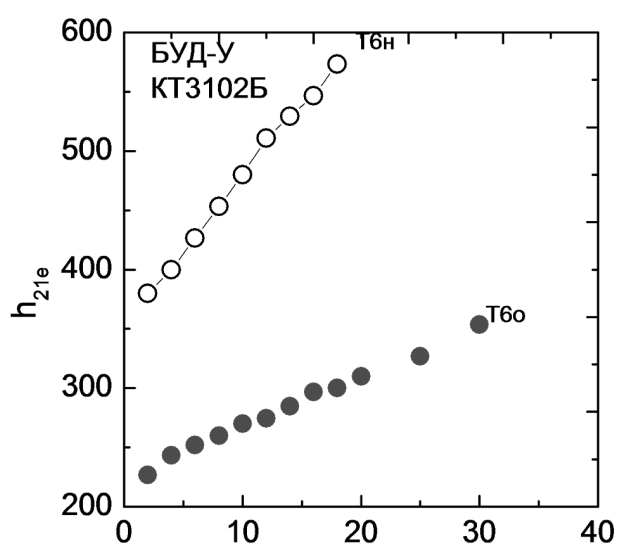

Fig. 7. The factor ideality of used transistors

Positively charged copper ions form couples with negatively charged acceptor ions-boron and consequently a portion of the dissolved copper ions is stationary. The effective diffusion coefficient of copper in the silicon alloyed with boron [5] is:

$$
D_{\text {eff }}=3 \times 10^{-4} \exp \frac{(-2090 / T)}{1+2.584 \times 10^{-20} \exp (4990 / T)\left(N_{a} / T\right)}, \quad \mathrm{cm}^{2} \mathrm{~s}^{-1} .
$$

In p-type silicon the copper forms copper-copper pairs. The analysis shows that they are formed between atom in node and atom in internode. The time of the dissociation of the copper-copper couples is:

$$
\tau_{C u-\text { pair }}=1.47 \times 10^{-10} \exp \left(\frac{1.02 \pm 0.07 \mathrm{eV}}{k_{B} T}\right), \mathrm{s} .
$$

This equation gives time constant from 16 days to 9.5 years. The average value is about 8 months. Process with such a constant can be completely decisive for the slow degradation of silicon diodes and transistors. The lifetime in $\mathrm{n}$-and $\mathrm{p}$-silicon varies in a different manner depending on copper concentration. Influence of lifetime and its changes on volt-ampere characteristics of p-n junctions is well described by the model of Stafeev $[6,7]$.

From the analysis of the above models, combined with the experimentally obtained data, it is built a theoretical model for the slow degradation of diodes and transistors, which are a major elemental base in electron equipment for the management and control of NPP. For diodes and transistors the analytical 
forms of the model are represented by equations (16) and (17):

$$
\begin{gathered}
\beta=(0.066 \pm 0.042) \times \text { Years }+(1.49 \pm 0.238), \\
h_{21}=(-8.006 \pm 5.033) \times \text { Years }+(162.758 \pm 28.473),
\end{gathered}
$$

and graphically presented in Figs 8 and 9, respectively.

Figure 10 shows that the increase of the ideality factor $\beta$ of tested diodes is in the range 1.15-1.8. A similar picture was seen in the relative change of the transmission coefficient of $h_{21}$ of the transistors, which is shown in Fig. 11.

The observed changes in the studied parameters can be explained noncontradictory with the assumption that they are mainly due to the high solubility and diffusion capacity and the specific behaviour of residual copper atoms in silicon plates from which the tested specimens are made. On the basis of the known values of the coefficient of diffusion of cooper into silicon and the dynamics of the formation and destruction of couples of cooper ions and precipitates, time constants of the degradation processes were evaluated of about 15 days to more than 9 years.

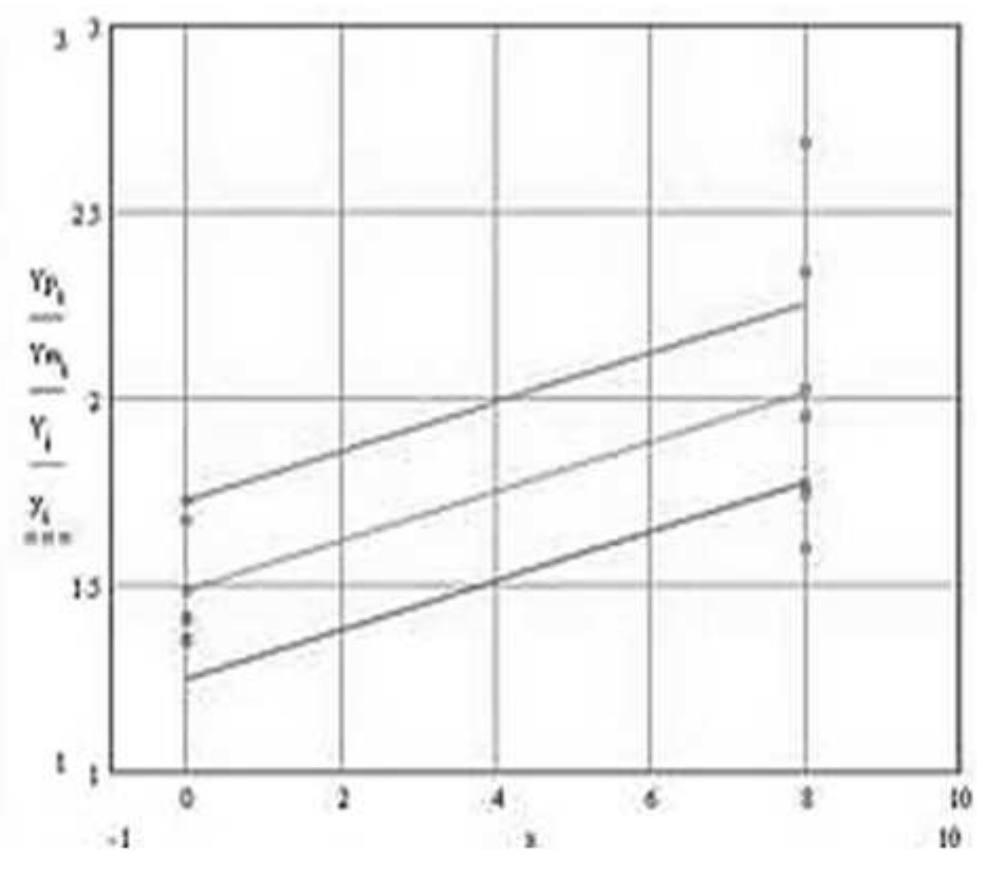

Fig. 8. Graphical presentation of diodes aging as a function of $(Y=\beta, x=$ years $)$ 


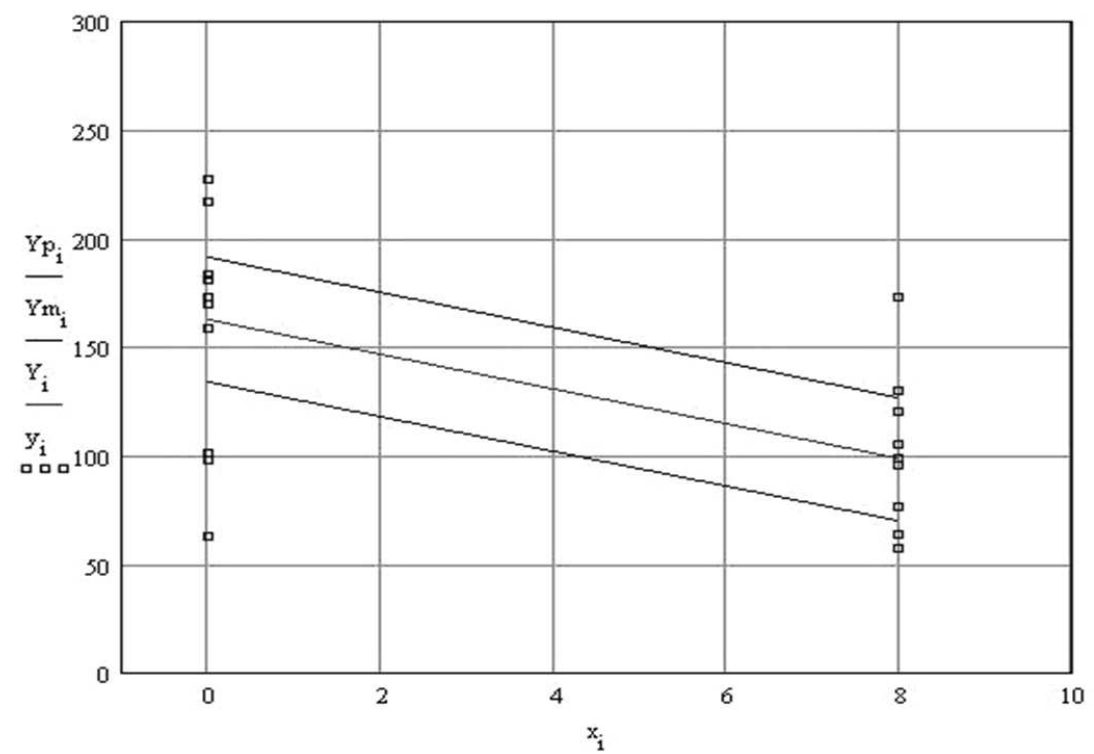

Fig. 9. Graphical presentation of transistors aging as a function of operating time $\left(Y=h_{21}, x=\right.$ years $)$

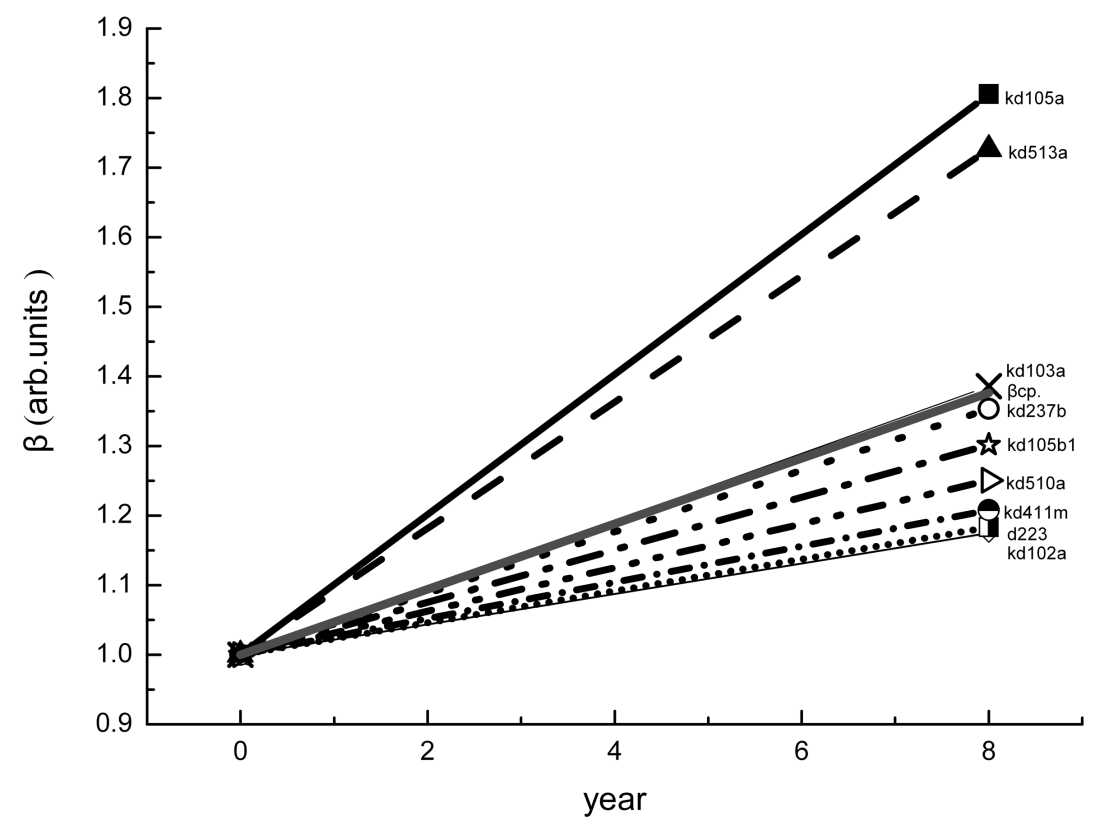

Fig. 10. Arbitrary changes of $\beta$ of tested diodes 


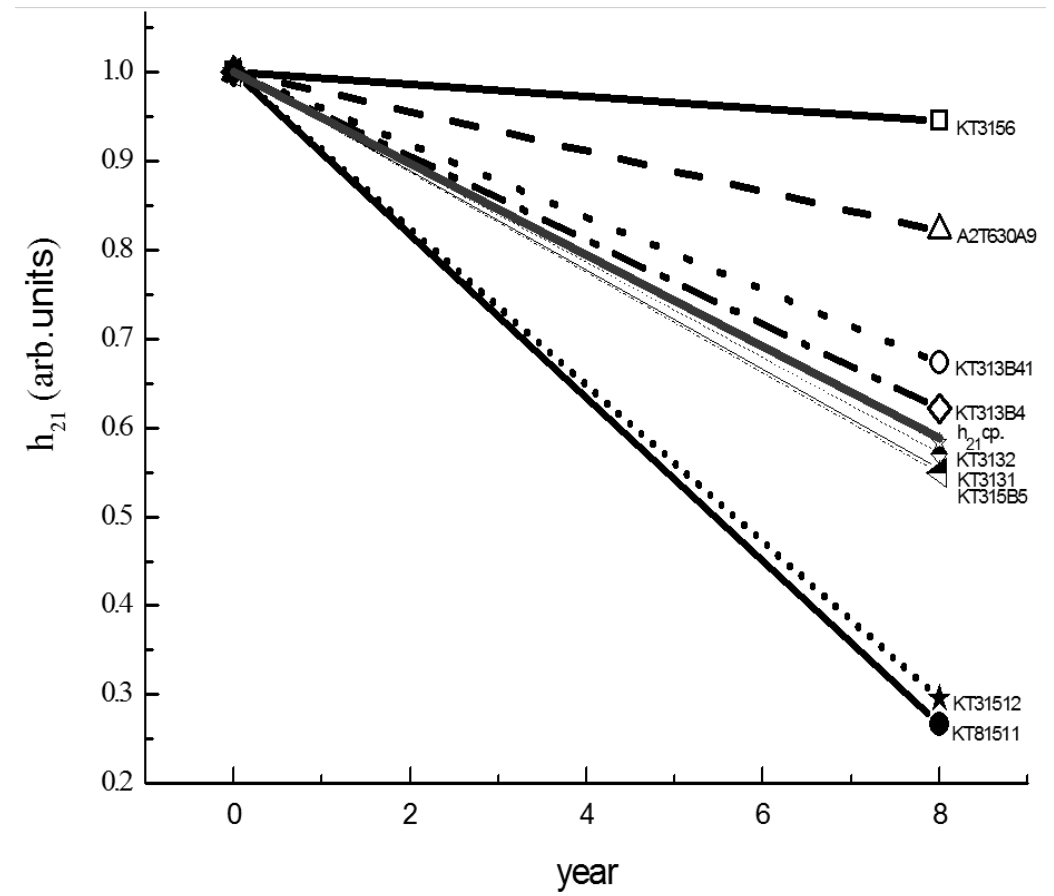

Fig. 11. Arbitrary changes of $h_{21}$ of tested transistors

\section{ELECTRO-CONTACT SYSTEMS [8]}

The second main group of elementary base of electronic equipment of NPP are electro-contact systems, for which are studied: ampere-volt features of couple of pairs of contacts from each contactor for determination of contactor resistance $R_{k}$; micro-hardness $H$ of contact surfaces of unused and used contactors independently of light and dark areas where they are detached; ampere-volt characteristics for defining of surface-resistance $R_{s}$, separately of light and dark regions of contact areas (of unused and used contactors).

\subsection{Measurement and analysis of contact resistance of contacts in mechanical contactor systems}

The first and most important performance parameter for all contactor devices with mechanical metal contacts (or metallurgical contacts, such as thermocouples and some other control devices) is their contact resistance $R_{k}$. It is extremely sensitive to any degradation processes and its quantitative values are an indicator of whether the contactors have entered a phase of ageing or are in conditions of normal operation. 


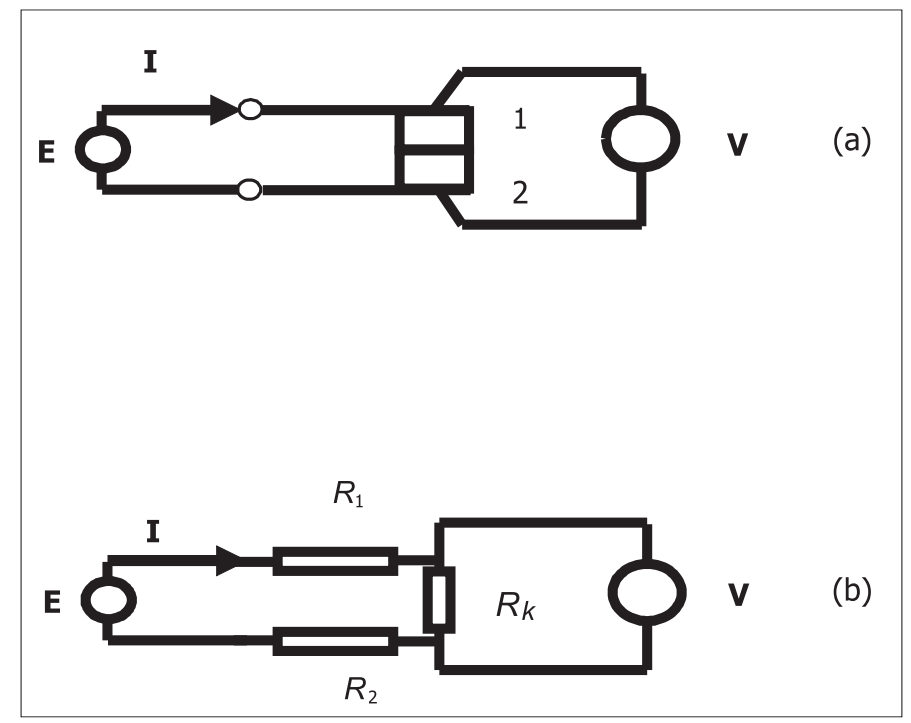

Fig. 12. Plan of a four electrod methodology:

(a) schematic; (b) equivalent

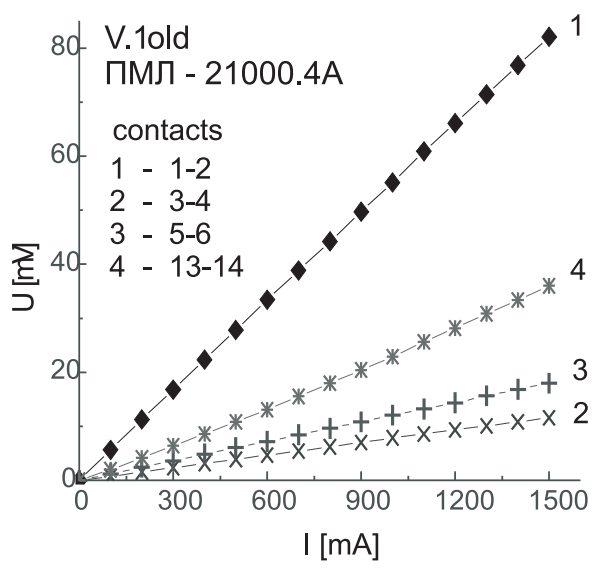

(a)

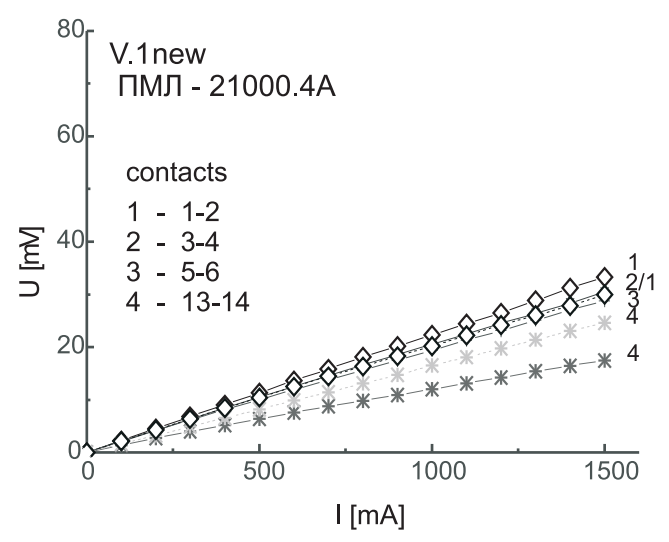

(b)

Fig. 13. Volt-ampere characteristics of:

(a) old contacts; (b) new contacts 
Characteristic for the beginning of this degradation process is the relative increase of the contact resistance between the contacting pads, measured in closed contact. For its determination a method is used for comparative measurement of the residual potential difference between the contact spots in operating position of the used and unused contactors of the same type. Measurement is done by a four electrode methodology - a modified two probe method, Fig. 12(a). According to the equivalent scheme shown in Fig. 12(b) the residual potential difference $U_{12}$, registered by the voltmeter, is determined by cross resistance of the contacts. Elements $R_{1}$ and $R_{2}$ represent the electric resistance of the leading rails and $R_{k}$ - the aggregate contact resistance of the two contact spots $\left(R_{k}=R_{k 1}+R_{k 2}\right)$ :

$$
R_{k}=R_{k 1}+R_{k 2}=U_{12} / I \text {. }
$$

The potential probes are located in close proximity to the contact spots, so that the measured potential difference $U_{12}$ reads the voltage drop mainly on a high resistive component of the near contact layers, designated with $R_{k}$. Reference measurements of used and unused contactors of the same type replace the absolute geometric calibration. This paragraph describes measurements done to study the contact resistance of mechanical contactors. Described measurements are non-destructive and are carried out under normal pressure on contacts: of an activated contactor for normally open contacts and of nonactivated contactor for normally closed contacts and in currents comparable to the nominal ones for operation. After summarizing the results, on selected representative contact couples volt-ampere features are modelled. Furthermore, again on the selected contacts electric probe measurements are made, as well as a study of microhardness and position by an X-ray microprobe. Potential probes are placed as close as possible to the contact spots, so that the measured potential difference $U_{12}$ reads the voltage drop mainly on the high resistive component of the near contact layers, denoted by $R_{k}$.

Described measurements are nondestructive and conducted under standard pressing on contacts: contactor activated for normal open contacts and nonactivated for normal closed contacts at nominal current operation, Fig. 13(a, b).

\subsection{Measurements of microhardnes}

The study of microhardness of contactor spots is performed using microhadnessmeter. Measurements are conducted at room temperature. The pressure on the indenter $P$ could be selected in the interval $0.5-200 \mathrm{~g}$. The average

Engineering Sciences, LVII, 2020, No. 3 
microhardness is calculated by software for a given loading:

$$
H=A P / d^{2},
$$

where the value of the constant $A$ depends on the type of the selected indenter. At the same time the deviations from the average value of $H$ are calculated, as well as the depth of indenter penetration into the specimen $d$. In order to examine the profile of change in the microhardness as a function of the penetration depth of indenter, measurements are carried out over a wide range of load by at least such that it is possible to measure the fingerprint, to one in which to reach a steady characteristic for a given material microhardness.

The method may also be used in carrying out the phase analysis. If the specimen is multiphase and different phases are large enough to be indented, microhardness of each phase can be measured. Since it depends on the composition, structure and type of processing the material, the resulting information [3] appears useful for the study of the complex system and a contactor, in particular for their residual resource. This is shown in Fig. 14.

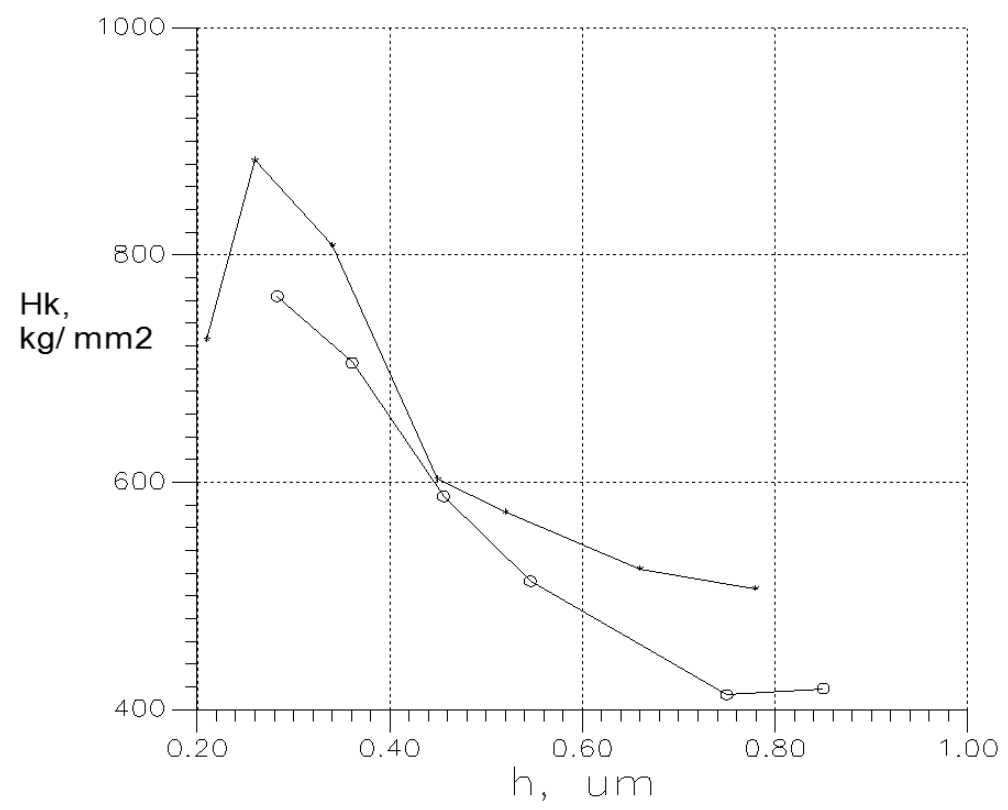

Fig. 14. Microhardness dependence on the penetration depth of indenter

Residual resource of contactor elements is evaluated by comparing the slopes of the volt-ampere features of working and non-working patterns, and 
causes of degradation phenomena are determined by measurements of microhardness and phase analysis of alloys, constituting the contact spots. For the degradation determination it is used: (i) linear schedule in two points determines the rate of degradation for a period of 8 years; (ii) for a basic mechanism of aging the diffusion of the metal atoms in the alloy is considered in less percentage as the formation of microphases and destruction of the contactor alloy. As the main factors facilitating this process are determined: temperature, operating current and time. Almost all unused contacts have linear volt-ampere features and close contact resistances of several $\mathrm{m} \Omega$. Large part of the used contactors is with linear volt-ampere characteristics but with higher contact resistances and they have additional working time. The average characteristics of both type are shown in Fig. 15.

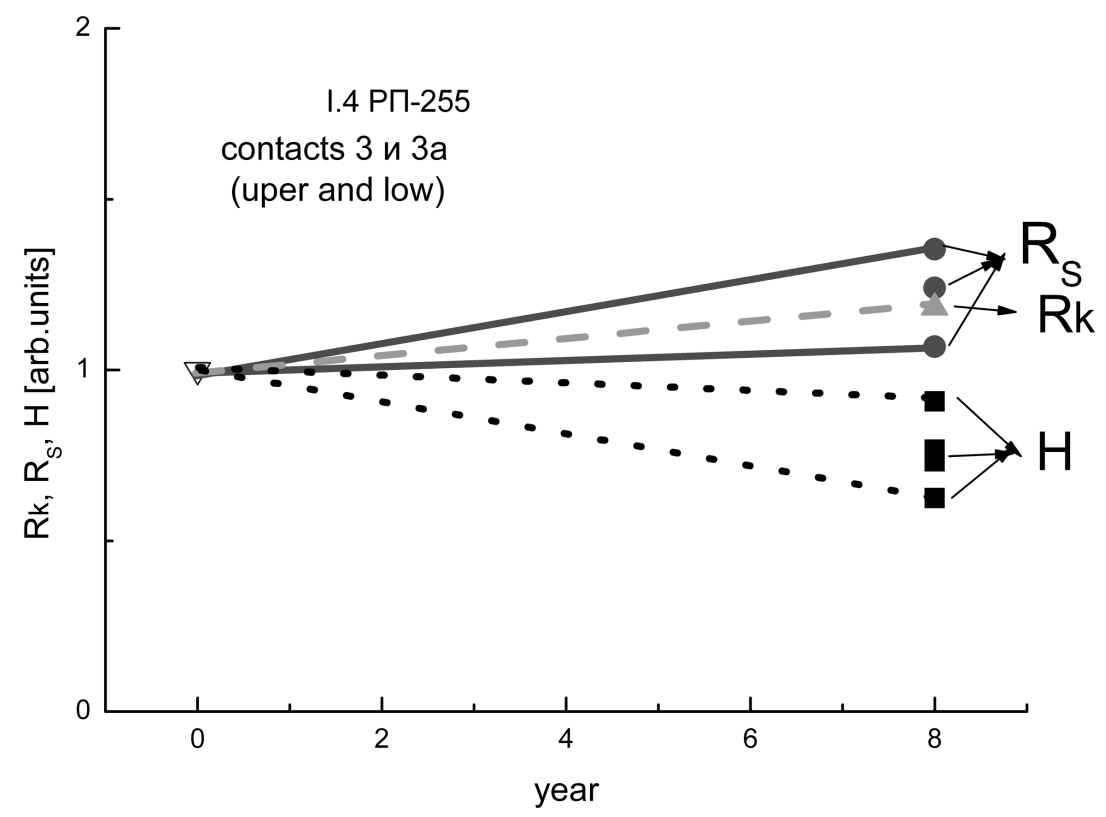

Fig. 15. Relative degradation changes of the three investigated parameters for unused and used contactors

Similarities in the relative degradation changes of the three investigated parameters and significantly greater number of contact sites of contactors in many design variations have given the reason and a possibility to find a unifying idea to explain the underlying processes, causing degradation of contactors during operating process. 


\section{INSULATION MATERIALS [9]}

Electronic equipment management and control of various systems of power Units No 5 and 6, comprising insulating material, are tested for the presence of degradation processes, occurring in continuous use in background controlled in sense of radiation according to safety standards in NPP Kozloduy at different weather conditions. Selected systems are operating in continuous mode about 10 years and they have taken separate blocks containing different dielectric materials (paint coatings, substrates for printed circuit boards, cable insulation and electrical protection elements). There are measured dielectric permittivity $\varepsilon$, dielectric losses factor $\operatorname{tg} \delta$, and specific resistivity $\rho$ of used dielectric materials and insulators for cables of electronic equipment of NPP. The resulting values are averaged over all measured patterns and compared with available tabulated values for the specific insulating materials. The comparison is made with the table data, since it is well known that in the dielectric material degradation changes were observed in time without being subject to any real exploitation. The measurement of main parameters of the identified models is performed and analyzed by a theoretical model according to which upon degradation of dielectric and insulation materials the electrical conductivity increases at operating voltages. This is related to a change in the polarization mechanism of dielectric dipole structure or the occurrence of double injection.

\subsection{Volt-ampere characteristics}

It is examines the linearity of the volt-ampere feature of the tested specimens, as the voltage is changed from about 200 to $1000 \mathrm{~V}$ with a step of 50 $\mathrm{V}$. If the ratio current/voltage is not preserved, these are symptoms of several

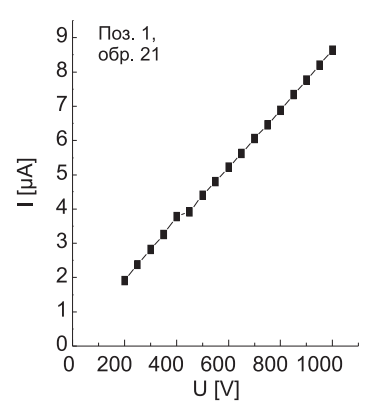

(a)

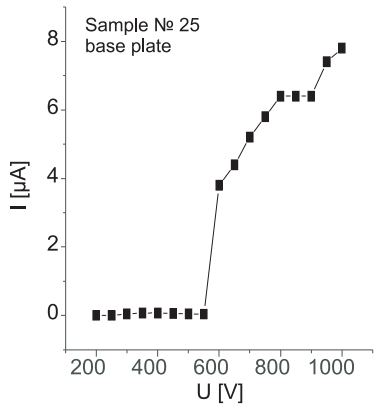

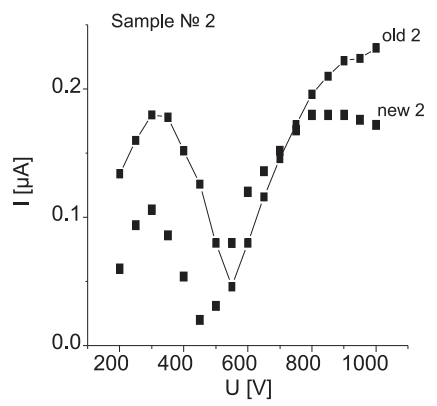

(c)

Fig. 16. Three types of volt-ampere characteristics of dielectric materials 
possible processes, namely: a change of the polarizing machanism, forming conductive cords, or the occurrence of a double injection in a dielectric, which can be considered as wideband semiconductor. Data obtained from three types are shown in Fig. 16(a, b, c):

a) the majority (about $80 \%$ ) has no strong deviations from the linear character;

b) in another group (about 10\%) there are horizontal initial sections in the volt-ampere features which are offset relative to zero of the abscissa. This is an indication of the beginning of sutface leakages;

c) in a third group (about 10\%) sections with a negative resistance are observed at about $500 \mathrm{~V}$, which suggest degradation processes associated with a double injection.

For the specimens of three types of defined shape and size it is also possible to determine independently the dielectric loss factor by the separate measurement of the two components of the conductivity and by bridge allows operation in two modes: (i) direct measurement of the dielectric loss factor; (ii) separate measurement of the capacity (for the determination of relative permittivity) and the active impedance (for possible calculating the dielectric loss factor).

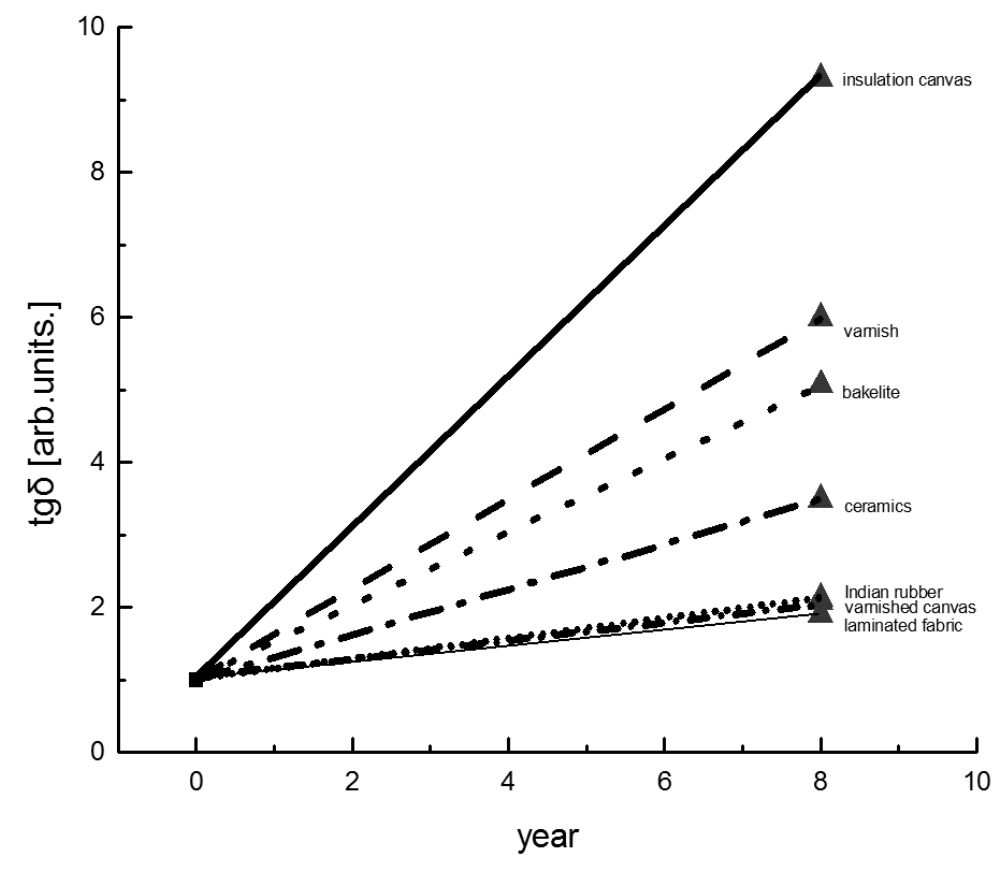

Fig. 17. Grouping of dependencies of dielectric losses of different materials as a function of operating time 
Preliminary comparison of the measured values with same tabular data for the discussed materials shows predominantly falling within the tolerances as shown in example increase of dielectric loss factor.

The fact that the relative degradation changes of the basic parameters of such a large group of diverse materials (for example it is shown in Fig. 17 for $\operatorname{tg} \delta$ ) have similar numerical intervals shows some community of processes causing degradation. This and the results of the study of the previous two groups of components (diodes and transistors and contactors) allow the construction of a general explanation of the observed changes in the parameters of electronic components and materials in the process of operation, Table 1.

Table 1

\begin{tabular}{|c|c|c|c|c|c|}
\hline No & \multicolumn{2}{|c|}{ Experimental data } & \multirow{2}{*}{$\begin{array}{c}\text { Sample } \\
\text { materials }\end{array}$} & \multicolumn{2}{|c|}{ Table data } \\
\hline & $\varepsilon$ & $\operatorname{tg} \delta$ & & $\operatorname{tg} \delta$ \\
\hline 3 & $2.9-3$ & 0.03 & vinylite & $3.2-4$ & $0.01-0.05$ \\
\hline 14 & 3.6 & 0.13 & insulation canvas & $3-4.5$ & $0.02-0.30$ \\
\hline 15 & 7 & 0.15 & bakelite & $5-12$ & $0.02-0.50$ \\
\hline 16 & 14 & 0.006 & ceramics & & $0.0005-0.025$ \\
\hline
\end{tabular}

\section{CONCLUSIONS}

From the conducted studies, it is clear that the main degradation processes in the three groups of elements for an extended period of time are slow, do not lead to a hopping change in basic parameters and to catastrophic failures. This gives ground to suggest a common diffusion model, which is limited by the following: (i) in electronic components containing a p-n junction, diffusion of residual copper atoms that are accumulated in the area of a spatial charge is performed under the influence of the electric field and the local temperature, creation of shunt channels; (ii) in the contactor systems whose contact surfaces are made of metal alloys under the influence of incresed temperature decomposition of a homogeneous alloy starts. Conditions are created for diffusion of individual atoms to the surface, microphases of homogeneous atoms are formed and modify the contact resistances; (iii) in the course of time in the insulating materials the mechanisms of polarization are changed, double bonds and dipoles are disrupting, leading to the release of carbon atoms. The latter diffuse at elevated temperature and form conductive cords, which amend the dielectric losses and specific resistance of the materials.

Therefore, the total physical model of slow degradation in electronic systems can be built on diffusion processes of free atoms under the influence of 
local temperature and electric field. These processes are uncontrollable, their speed is slow, and the time of change of the resource can be predicted by using the developed methods.

\section{REFERENCES}

[1] S. Popov, Degradation model of red GaP LEDs, Phys. Stat. Solidi (a) (1984) 81669.

[2] M. Seibt and K. Graff, Characterization of has-forming precipitates in silicon, J. Appl. Phys. (1988) 634444.

[3] A. Popov And N. Naydenov, Study and analisys of degradation processes in the electronic equipment operating in Kozloduy NPP, J. Optoel. Adv. Mater. (2005) 7 (1) 321.

[4] A. A. Istratov et al., Intrinsic diffusion coefficient of interstitial copper in silicon, Phys. Rev. Lett. (1988) 811243.

[5] A. A. Istratov et al., 1988 The dissociation energy and the charge state of a copper-pair in silicon, Appl. Phys. Lett. (1988) 72474.

[6] V. I. STAFEEV, Influence of the resistance semiconductor materials on volt-ampere characteristic of diodes, J. Tech. Phys. (1958) 281631.

[7] V. I. Stafeev, Modulation of diffusion length as a new principal working method of semiconductor devices, Solid State Physics (1959) 1841.

[8] N. Naydenov and A. Popov, Pre- and post-servise microhardness measurements of electrical contacts operating at Kozloduy NPP, Mater. Sci. \& Eng. B (2006) 132247.

[9] N. Naydenov and A. Popov, Study of degradation processes in dielectric materials in electronic control equipment operated in Kozloduy NPP, Conf. Proc. of J. Amer. Inst. Phys. (2007) 89975.

Received July 21, 2020

Engineering Sciences, LVII, 2020, No. 3 